\title{
Three-dimensional electric field mapping of an electrically biased atom probe needle using off-axis electron holography
}

Fengshan Zheng ${ }^{1}$, Vadim Migunov ${ }^{1,2}$, Jan Caron ${ }^{1}$, Hongchu Du ${ }^{1,2}$, Giulio Pozzi ${ }^{1,3}$ and Rafal E DuninBorkowski $^{1}$

1. Ernst Ruska-Centre for Microscopy and Spectroscopy with Electrons and Peter Grünberg Institute, Forschungszentrum Jülich, 52425 Jülich, Germany

2. Central Facility for Electron Microscopy, RWTH Aachen University, 52074 Aachen, Germany

3. Department of Physics and Astronomy, University of Bologna, Viale Berti Pichat 6/2, 40127 Bologna, Italy

Atom probe tomography (APT) involves the detection of ions that have been field evaporated from a sharp needle-shaped specimen [1]. A precise experimental knowledge of the three-dimensional electric field outside the specimen, which influences the trajectories of the ions [2], promises to provide improved reconstructions of atomic positions. Off-axis electron holography provides direct access to the projected electrostatic potential within and around a specimen in the transmission electron microscope (TEM) [3]. Here, we use the technique to determine the electric field around a $\mathrm{C}$ fibre atom probe needle, which is electrically biased in situ in the TEM using a scanning tunnelling microscopy (STM)-TEM specimen holder [4].

Figure 1a shows a low magnification bright-field TEM image of the setup, which is similar to that used for field evaporation during APT experiments. The distance between the Au counter-electrode and the atom probe needle is approximately $1 \mu \mathrm{m}$. Figure $1 \mathrm{~b}$ shows a contoured electron holographic phase difference image obtained by subtracting a phase image recorded without an electrical bias applied to the needle from a phase image recorded at an electric bias of $+40 \mathrm{~V}$, in order to remove the mean inner potential contribution to the phase. The strength of the projected electric field around the needle can be calculated from the gradient of the phase difference image, as shown in Fig. 1c. It is highest at the apex region (approximately $100 \mathrm{MV} / \mathrm{m} . \mathrm{m}$ ). The asymmetry in the maps of electric field and phase results from the influence of the so-called perturbed reference wave (PRW) [5].

We are presently developing a numerical model-based approach for reconstructing the threedimensional charge density distribution inside a specimen [6] from a tilt series of phase images. This approach allows the incorporation of a priori information, such as the shape of the object (i.e., the region in which charge can be located), as well as the use of a confidence mask to exclude regions containing artefacts from the analysis. The method is also unaffected by the PRW and the presence of charges outside the field of view. The three-dimensional electric field can be reconstructed from the resulting charge density distribution with fewer artefacts and improved signal-to-noise ratio than by applying conventional backprojection-based tomographic reconstruction techniques directly to the original recorded phase images.

Figure 2 shows two representative contoured electron holographic phase images extracted from a tomographic tilt series. The tilt increment was $4^{\circ}$ and the tilt range was $+48^{\circ}$ to $-52^{\circ}$. At each specimen tilt angle, the mean inner potential contribution to the phase was removed by subtracting a phase image recorded without a bias voltage [7].

References:

[1] T.F. Kelly, D.J. Larson, Annual Review of Materials Research. 42 (2012) 1-31.

[2] T.F. Kelly, Microscopy and Microanalysis. 23 (2017) 34-45.

[3] H. Lichte, M. Lehmann, Reports on Progress in Physics. 71 (2008). 
[4] K. Svensson, Y. Jompol, H. Olin, E. Olsson, Review of Scientific Instruments. 74 (2003) 4945.

[5] G. Matteucci, G.F. Missiroli, M. Muccini, G. Pozzi, Ultramicroscopy. 45 (1992) 77-83.

[6] J. Caron, Ph.D. Thesis, RWTH Aachen University, 2017.

[7] The authors acknowledge the European Union for funding through the Marie Curie Initial Training Network Grant No. 606988 under FP7-PEOPLE-2013-ITN).
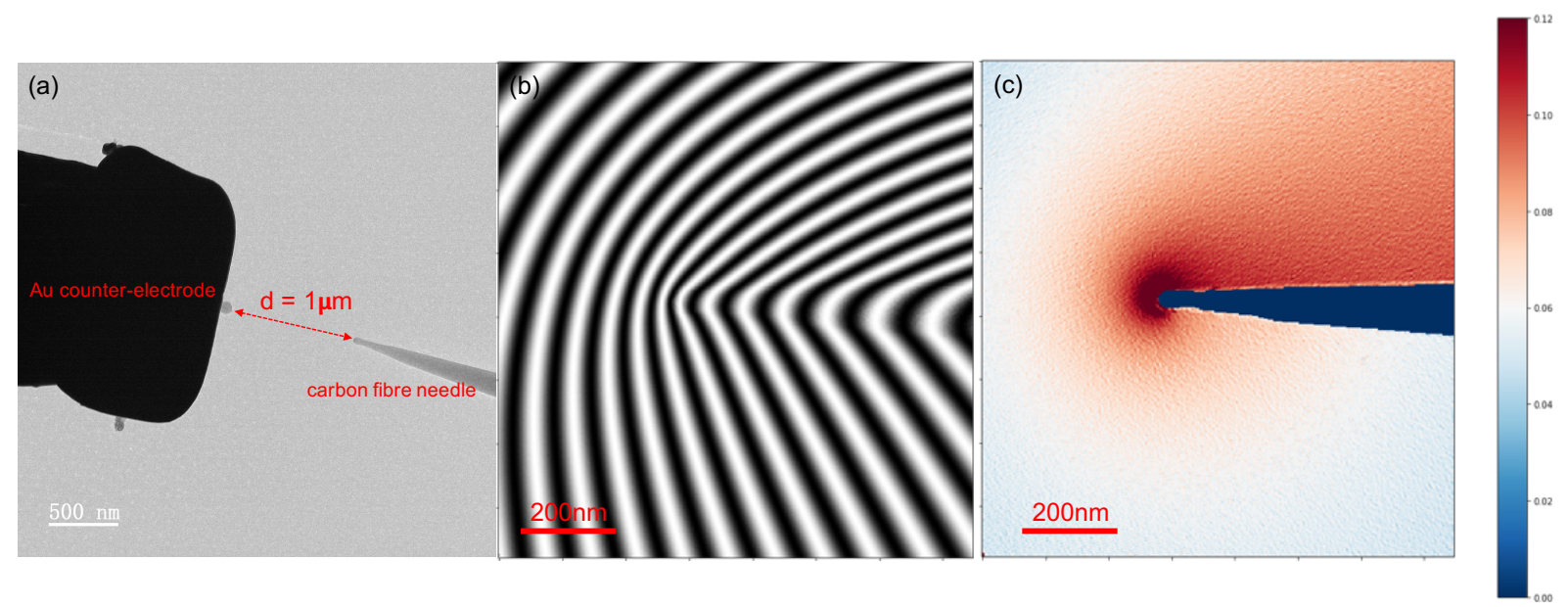

Figure 1. Off-axis electron holography of an electrically biased C fibre atom probe needle: a) geometry of the experimental setup; b) Contoured phase difference image obtained by subtracting a phase image recorded without an electrical bias applied to the needle (corresponding to the mean inner potential contribution to the phase) from a phase image recorded at an applied bias voltage of $+40 \mathrm{~V}$. The contour spacing is $2 \pi$ radians. c) Magnitude of the projected electric field outside the needle inferred from the phase difference image shown in b). The blue region marks the interior of the needle.

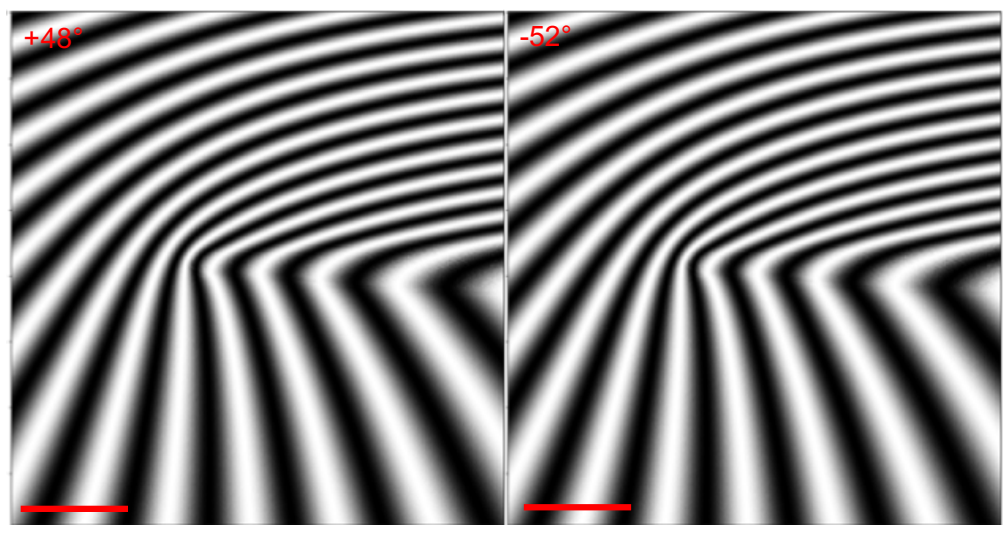

Figure 2. Two contoured phase images extracted from a holographic tomographic tilt series (left: $+48^{\circ}$; right: $-52^{\circ}$ ) with the needle electrically biased at $+40 \mathrm{~V}$. The mean inner potential contribution to the phase has been removed from each image. The contour spacing is $2 \pi$ radians. The scale bar is $200 \mathrm{~nm}$. 